\title{
Preparation of a Spironaphthoxazine Moiety-Containing Methyl Cellulose and Photoregulation of Its Properties
}

\author{
Kenichiro AraI, ${ }^{\dagger}$ Takeshi Ohyama, and Yasutada Shitara
}

Faculty of Engineering, Gunma University, Kiryu, Gunma 376, Japan

(Received March 3, 1997)

\begin{abstract}
KEY WORDS Spironaphthoxazine / Methyl Cellulose / Reversible Isomerization / UV spectrum / Duarability / Contact Angle /
\end{abstract}

Photoregulation of properties of polymers containing photochromic moieties has been the subject of many investigations. We have investigated cellulose derivatives containing photochromic moieties. ${ }^{1-6}$ In the previous study, we prepared the photochromic spiropyran moietycontaining methyl cellulose (SP-MC) and the spiropyran moiety in SP-MC was confirmed to be isomerized reversibly under alternating irradiation with UV and visible light, both in solution and in film. ${ }^{6}$ Furthermore, the solubility of SP-MC in benzene and the contact angle of the SP-MC film with water were reversibly regulated under alternating irradiation with UV and visible light. However, repeated cycles of the alternating irradiation with UV and visible light resulted in considerable fatigue of the spiropyran moieties over up to 20 cycles. In the present experiment, a spironaphthoxazine (SO) moiety containing methyl cellulose (SO-MC) was prepared and the reversible changes in the properties of the SO-MC under irradiation with UV light and subsequently with visible light, as well as the isomerization behavior of the SO moiety in the derivatives, were examined; the spironaphthoxazine was reported to be isomerized from the colorless closed ring form to the colored open ring form under irradiation with UV light (Scheme 1) and to have much greater durability than spiropyran moiety for the repeated irradiations with UV and visible light. ${ }^{7}$

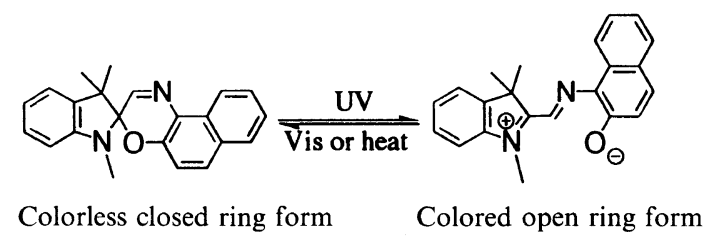

Scheme 1. Photoisomerization of spironaphthoxazine.

\section{EXPERIMENTAL}

\begin{abstract}
Materials
1- $\beta$-Carboxyethyl-3,3-dimethylspiro[indolino-2,3'naphth[2.1-b](1.4)oxazine] $(\mathrm{SO}-\mathrm{COOH})$ was prepared according to the method reported by Ono and Osada ${ }^{8}$ by the treatment of 1,3,3-trimethylindolenine with 3iodopropionic acid to obtain $1-\beta$-carboxyethyl-2,3,3-trimethylindolenium iodide (CE-TMI-I), and subsequent treatment of the CE-TMI-I with 1-nitroso-2-naphthol in the presence of trimethylamine. The product was
\end{abstract}

identified by ${ }^{1} \mathrm{H}$ NMR, IR, and melting point (ref, 186 $187^{\circ} \mathrm{C}$; found, $\left.186.5-187.5^{\circ} \mathrm{C}\right)$. Commercially available methyl cellulose (Wako Pure Chemicals Ind., $100 \mathrm{cP}$ ) with a degree of substitution (DS) of 1.52 was dehydrated in vacuo over diphosphorus pentaoxide. Tetrahydrofuran (THF) was dried over molecular sieves $3 \mathrm{~A}$. All other reagent-grade chemicals were used without further purification.

\section{$S O-M C$}

Methyl cellulose $\left(0.91 \mathrm{~g}, 5.0 \times 10^{-3} \mathrm{~mol}\right.$ of anhydroglucose units) and SO-COOH $\left(3.87 \mathrm{~g}, 1.0 \times 10^{-2} \mathrm{~mol}\right)$ were dissolved in THF $(80 \mathrm{ml})$ and then $N, N^{\prime}$-dicyclohexylcarbodiimide $\left(2.06 \mathrm{~g}, 1.0 \times 10^{-2} \mathrm{~mol}\right)$ and $p$-dimethylaminopyridine $\left(0.24 \mathrm{~g}, 2.0 \times 10^{-3} \mathrm{~mol}\right)$ were added. The mixture was kept at $5^{\circ} \mathrm{C}$ for $2 \mathrm{~h}$ and then at $25^{\circ} \mathrm{C}$ for $96 \mathrm{~h}$ with stirring; the antecedent reaction at lower temperature favors dehydration-dimerization of SO$\mathrm{COOH}^{9,10}$ and then the dimer reacts with methyl cellulose at $25^{\circ} \mathrm{C}$ to give SO-MC (Scheme 2). The reaction mixture was filtered to remove insoluble material and then the filtrate was poured into methanol. The precipitate was recovered and purified by reprecipitation from THF solution three times into methanol and once into $n$-hexane.

The degree of substitution (DS) by the SO moiety of the SO-MC obtained was determined by elemental analysis: C, 64.8; H, 6.0; O, 24.7; N, 4.50; DS =0.94.

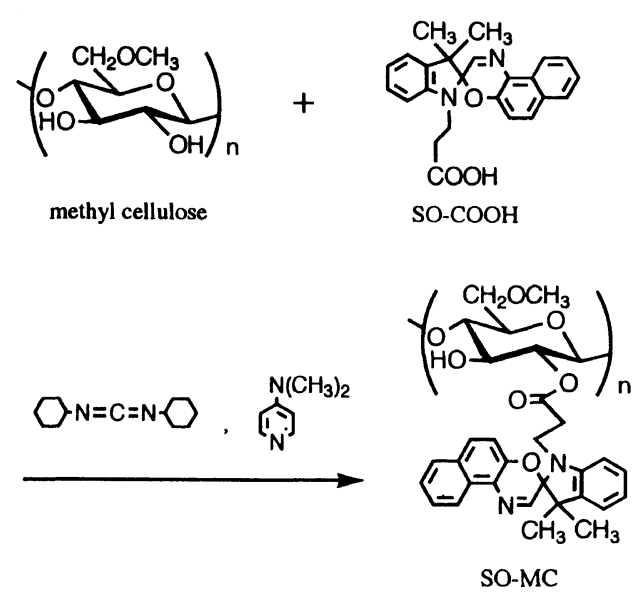

Scheme 2. Preparation of SO-MC.

\footnotetext{
† To whom correspondence should be addressed.
} 


\section{Irradiation}

UV and visible light from a high-intensity projecting lantern (Ushio Electric Inc., Tokyo; 200W) were used for the irradiation of SO-MC in solution and in film using color filters (Toshiba UV-D36A and Toshiba O-55 for $\lambda=300-400 \mathrm{~nm}$ and $\lambda>550 \mathrm{~nm}$, respectively).

\section{SO-MC Film}

An SO-MC film with thickness of $10 \mu \mathrm{m}$ was prepared by casting from the SO-MC THF solution and dried in vacuo. The film was used for the examination of the isomerization behavior of SO moiety in the film and the changes in the contact angle of the film with water under irradiation with UV light and with visible light.

\section{$I R$ and $U V$ Absorption Spectra}

IR absorption spectra of SO-COOH, methyl cellulose, and SO-MC were obtained with a double-beam grating FT-IR spectrometer (Japan Spectroscopic Co., Tokyo; FT-IR 5300) using the $\mathrm{KBr}$ disk technique. UV absorption spectra of SP-MC in solutions and in film were obtained with a Hitachi 200 spectrometer.

\section{Contact Angle of SO-MC Film}

The contact angle of the SO-MC film with water was measured using a contact angle meter (Kyowa Kaimenkagaku Co., Ltd., Tokyo; CA-P) at ambient temperature.

\section{RESULTS AND DISCUSSION}

The presence of the SO moiety in SO-MC was confirmed in terms of IR absorption spectrum. An absorption peak around $3400 \mathrm{~cm}^{-1}$, which is assigned to the hydroxyl group, in the spectrum of methyl cellulose decreased with the introduction of SO moiety. An absorption peak at $3069 \mathrm{~cm}^{-1}$, which is assigned to aromatic $\mathrm{C}-\mathrm{H}$, and those at 810 and $750 \mathrm{~cm}^{-1}$, which is assigned to substituted benzene, were found in the spectrum of SO-MC instead. An absorption peak at $1745 \mathrm{~cm}^{-1}$, which is assigned to an ester group, was also found.

Coloration by the isomerization of SO-MC in common organic solvents, such as benzene, toluene, acetone, 2-butanone, pyridine, and 1,2-dioxane, under irradiation with UV light of $\lambda=300-400 \mathrm{~nm}$ was examined and the coloration to blue was observed in all the solvents. The colored form of SO moiety was unstable and the color disappeared only in a few seconds even in the dark. However, SO-MC dissolved in chlorinated solvents such as chloroform and dichloromethane was found exceptionally to color in reddish purple and the color was more stable in the dark, although the maximum absorbance obtained was lower than those in the common organic solvents. These results may reflect that the colored open ring form of SO moieties are stabilized, probably due to interaction of the open ring form with the chlorinated solvent molecules, although the details of the mechanism are not apparent.

Changes in the UV absorption spectra of SO-MC in solutions and in film under irradiation with UV light of $\lambda=300-400 \mathrm{~nm}$ and subsequently with visible light of $\lambda>550 \mathrm{~nm}$ were examined. Figure 1 shows changes

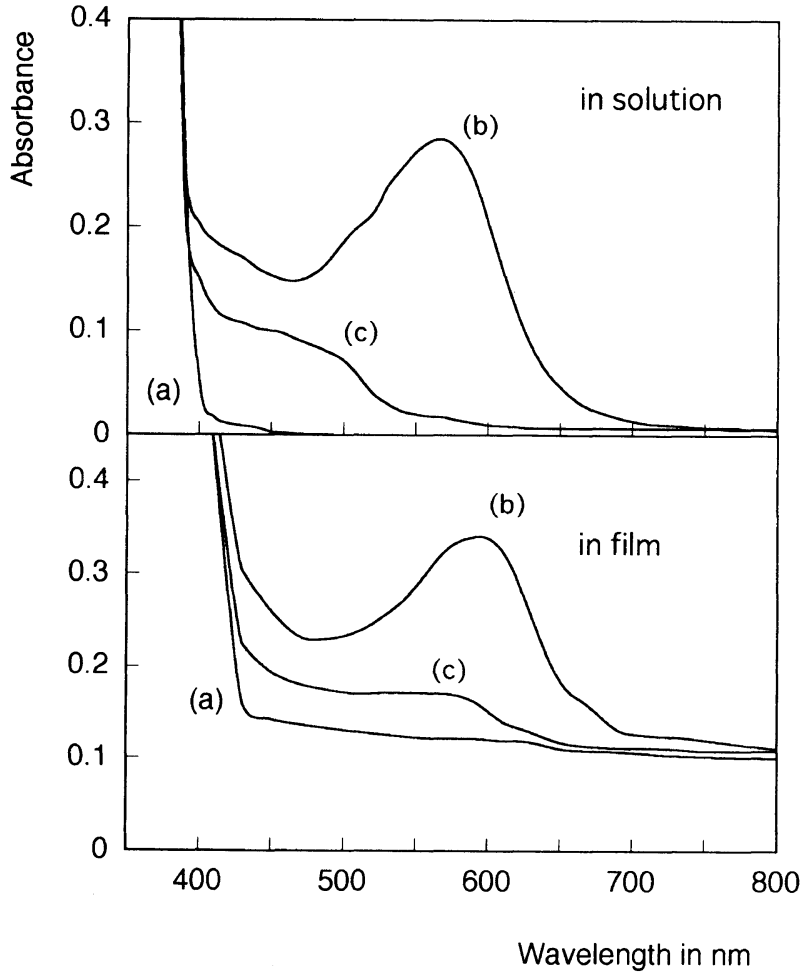

Figure 1. Changes in UV absorption spectra of spironaphthoxazine moiety-containing methyl cellulose (SO-MC, DS by spironaphthoxazine moiety $=0.94$ ) in dichloromethane solution (above) in film (below) under irradiation with UV light of $\lambda=300-400 \mathrm{~nm}$ and subsequently with visible light of $\lambda>550 \mathrm{~nm}$. (a) Before irradiation, (b) after irradiation with UV light for 1 and $5 \mathrm{~min}$ for the solution and for the film, respectively, and (c) after subsequent irradiation with visible light for 3 and $30 \mathrm{~min}$ for the solution and for the film, respectively. [SO-MC] in the solution $=0.21 \mathrm{~g} \mathrm{l}^{-1}$.

in the UV absorption spectra of SO-MC in dichloromethane solution (above) and in film (below) under irradiation with UV light and subsequently with visible light. For the dichloromethane solution, a new peak is found to appear at $563 \mathrm{~nm}$ under irradiation with UV light and is assigned to the open ring form. The peak height reached a maximum under irradiation with UV light for $1 \mathrm{~min}$ and the maximum absorbance is found to be lower than that of spiropyran moiety in SP-MC. ${ }^{6}$ The peak height decreases under irradiation with visible light, but the spectrum does not return to that before UV light irradiation. The difference in the peak height of SO-MC after irradiation with visible light from that before UV light irradiation is probably due to the difference in the isomerization equilibrium of the open ring form and closed ring form of starting SO-MC, which have been stored in the dark for a long time, from that of SO-MC irradiated with visible light in the room. In the second cycle of irradiation with UV light and subsequently visible light, the spectrum of SO-MC irradiated with visible light almost returned to that irradiated with visible light in the first cylce. For the film, a new peak is found at $591 \mathrm{~nm}$ in the UV absorption spectrum and the absorption peak also increases under irradiation with UV light with the irradiation time and then decreases under subsequent irradiation with visible light. However, much longer time is needed for the absorption peak of $\mathrm{SO}-\mathrm{MC}$ in film than for that of SO-MC in the solution to saturate under irradiation with UV light and then to 
decrease with visible light. This result proves that the isomerization of SO-MC moiety is also reversible in the film. The longer times needed for the isomerization of SO moiety in the film may be due to the spatial restrictions on the isomerizations of the closed ring form with a perpendicularly crossed planes structure to the planar open ring form one and then from the open ring form structure to the closed ring one, imposed by the polymer matrix. ${ }^{11,12}$

Since the absorption in the UV absorption spectra of SO-MC obtained in the solution and in the film under irradiation with UV light were found to decrease in the dark with the standing time, probably due to the thermal motion of SO moiety, the decrease rates in the absorption peak height under irradiation with visible light were compared with those in the dark.

Figure 2 compares the decrease rates of the absorbance of the colored SO-MC under irradiation with visible light, with those in the dark, for the absorption at $563 \mathrm{~nm}$ in dichloromethane solution (above) and for that at $591 \mathrm{~nm}$ in the film (below). Both in the solution and in the film, the absorbances obtained under irradiation with UV light were found to decrease considerably faster under irradiation with visible light than in the dark, although the decrease rates in the film are much slower than those in the solution. This result clearly shows that the irradiation with visible light markedly accelerates the isomerization of the open ring form SO moiety in SO-MC to the closed ring form one.

Alternating irradiation with UV and visible light at SO-MC in dichloromethane solution and in the film was repeated, and the changes in the absorbances in the UV absorption spectra of SO-MC with increasing number of repetitions were observed. Figure 3 shows the changes

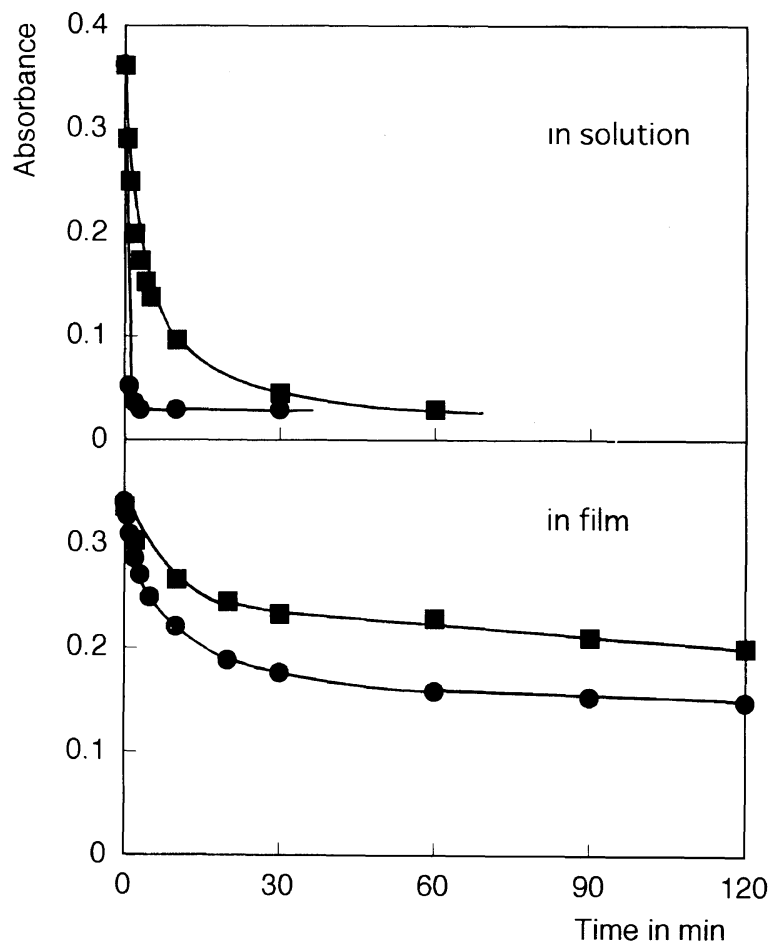

Figure 2. Changes in absorbances at 563 and $591 \mathrm{~nm}$ in UV absorption spectra of SO-MC in dichloromethane solution (above) and in film, respectively, after irradiation with UV light, under irradiation with visible light (O) and with standing in the dark ( $\square)$, with the time. in the absorbance at $563 \mathrm{~nm}$ in the spectrum of SO-MC in dichloromethane solution (above) and at $591 \mathrm{~nm}$ in the film (below) under repeated alternating irradiation, with number of repetitions. The irradiations with UV light were 1 and $5 \mathrm{~min}$ for the solution and for the film, respectively, and those with visible light were 3 and $30 \mathrm{~min}$ for the solution and for the film, respectively. Both in the solution and in the film, each absorption height is found to change reversibly with the repeated irradiations. In the solution, the absorbance at $563 \mathrm{~nm}$ obtained under irradiation with UV light decreases with the number of repetitions to about $70 \%$ of that after the first cycle after 20 repetitions. The absorbance after irradiation with visible light does not perfectly return to that after the first cycle and increases slightly with the number of repetitions. As the result, the difference between the absorbances after irradiations with UV light and with visible light becomes to about $50 \%$. The difference in the absorbance is still considerably larger than that of SPMC (about 30\%), previously reported. ${ }^{6}$ This decrease in the absorbance after irradiation with UV light and the increase in the absorbance after irradiation with visible light with the repetitions may reflect a fatigue of SO moieties in SO-MC under repeated alternating irradiations with UV light and visible light, although the details is not apparent. In the film, similar changes in the absorbance under repeated alternating irradiations with UV and visible light to those in the solution are found and the difference between the absorbances after irradiations with UV and after irradiation with visible light becomes to about $60 \%$, the value being considerably larger than that of SP-MC film (about 15\%), previously reported. ${ }^{6}$ It is worthy to note that the fatigue of SO

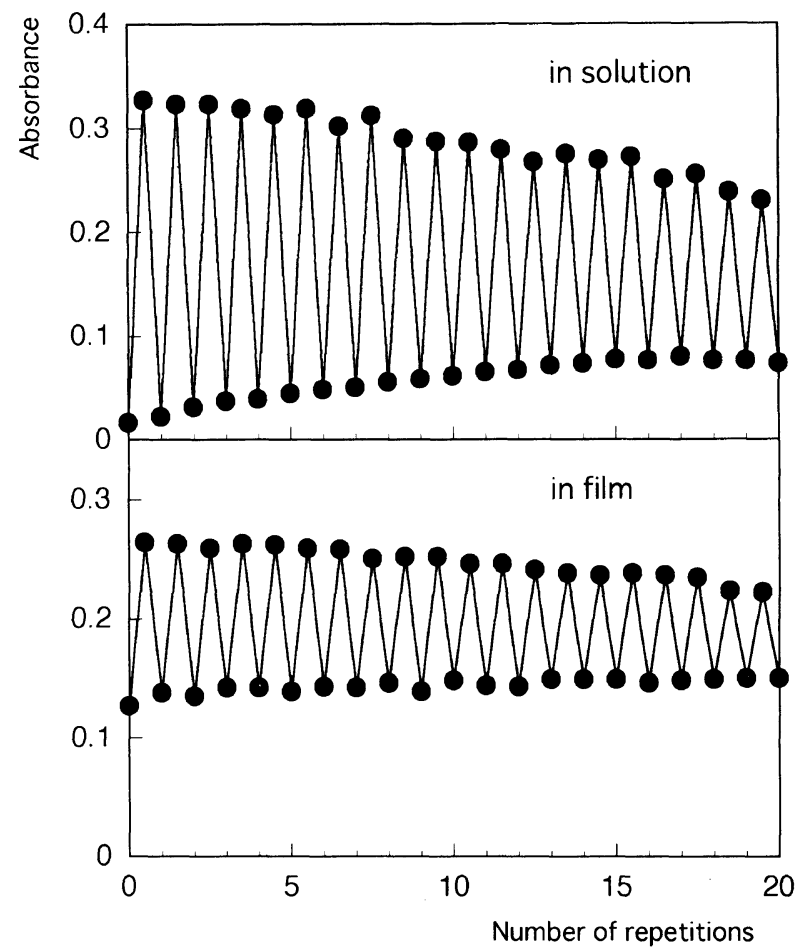

Figure 3. Changes in absorbances at 563 and $591 \mathrm{~nm}$ in UV absorption spectra of SO-MC in dichloromethane solution (above) and in film, respectively, under repeated alternating irradiations with UV light for 1 and $5 \mathrm{~min}$ for the solution and for the film, respectively, and subsequently with visible light for 3 and $30 \mathrm{~min}$ for the solution and for the film, respectively, with number of repetitions. 
moiety in SO-MC are considerably less than that of spiropyran moieties in SP-MC both in the solution and in the film, as expected, although the absorbance of SO moiety under irradiation with UV light itself is smaller than that of siropyran moiety.

Changes in the contact angle of the SO-MC film with water under irradiation with UV light and subsequently with visible light were examined. Figure 4 shows the changes in the contact angle, together with the change in absorbance at $591 \mathrm{~nm}$ in the UV absorption spectrum of the SO-MC film with irradiation time under the same condition. The contact angle is found to decrease under irradiation with UV light and then to increase with subsequent irradiation with visible light, with irradiation time. These changes in the contact angle correspond well to those in the absorbance at $591 \mathrm{~nm}$ in the UV absorption spectrum; The extent of the change in the contact angle of the SO-MC film is nearly the same in
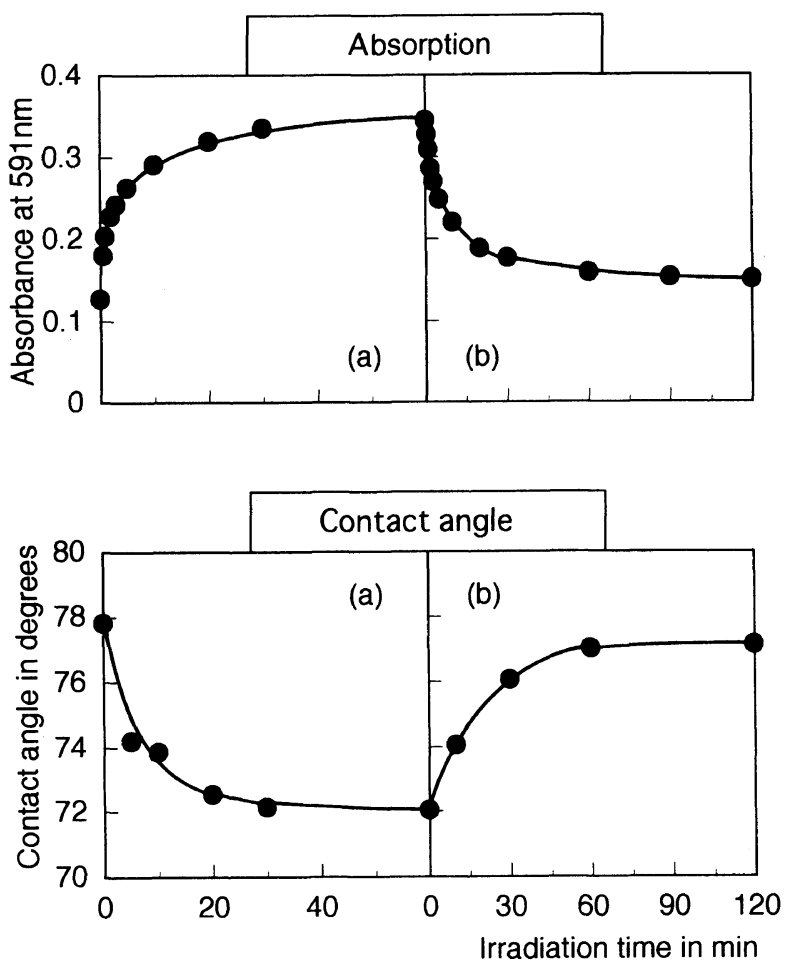

Figure 4. Changes in contact angle with water (below), together with those in absorbance at $591 \mathrm{~nm}$ in UV absorption spectrum (above) of SO-MC film under irradiation with (a) UV light and (b) subsequently with visible light, with irradiation time. spite of the lower changes in the absorbance as compared with those of the SP-MC film. This correspondence proves that the changes in the contact angle result from photoisomerization of the SO moiety to the more polar open ring form and from the open ring form to closed ring form in SO-MC.

\section{CONCLUSION}

SO-MC with DS by SO moiety of 0.94 was prepared by the treatment of methyl cellulose with SO-COOH and SO moieties in SO-MC were confirmed to undergo reversible isomerization under irradiation with UV light and subsequently irradiation with visible light in solution and in film. The repeated cycle of alternating irradiation with UV light and with visible light resulted in fatigue of the SO-moieties in SO-MC both in the dichloromethane solution and in the film, but the extent of the fatigue was considerably less than that of SP-MC, although the changes in the absorbance itself in the UV absorption spectrum of SO-MC was smaller than that of SP-MC. Contact angle of the SO-MC film with water were found to be reversibly regulated under irradiation with UV light and subsequently with visible light; the extent of the changes in the contact angle was nearly the same as that of SP-MC film in spite of the lower changes in the absorbance under irradiation with UV and visible lights. The regulation of the contact angle was deduced to result from the reversible isomerization of the SO moieties in SO-MC.

\section{REFERENCES}

1. K. Arai and H. Udagawa, Makromol. Chem., Rapid Commun., 9, 797 (1988).

2. K. Arai and H. Udagawa, Sen-i Gakkaishi (J. Soc. Fiber Sci. Tech. Jpn.), 46, 150, 491 (1990).

3. K. Arai and H. Satoh, J. Appl. Polym. Sci., 45, 387 (1992).

4. K. Arai, S. Sano, and H. Satoh, J. Mater. Chem., 2, 1257 (1992).

5. K. Arai and S. Sano, J. Mater. Chem., 4, 275 (1994).

6. K. Arai, Y. Shitara, and T. Ohyama, J. Mater. Chem., 6, 11 (1996).

7. M. Irie and K. Hayashi, Polym. Prep., Jpn., 34, 459 (1985).

8. H. Ono and C. Osada, US Patent 3562172 (1971).

9. H. G. Khorana, J. Chem. Soc., 2081 (1952).

10. J. C. Sheehan and G. P. Hess, J. Am. Chem. Soc., 77, 1067 (1995)

11. J. Kolc and R. S. Becker, J. Phys. Chem., 71, 4045 (1967).

12. R. Millini, G. D. Piero, P. Allegrini, L. Crisci, and V. Malatesta, Acta Cryst., C47, 2567 (1991). 